\title{
Effects of pitavastatin on plasminogen activator inhibitor-I in hyperlipidemic patients
}

This article was published in the following Dove Press journal:

International Journal of General Medicine

15 June 2012

Number of times this article has been viewed

\section{Shosaku Nomura \\ Takehito Taniura ${ }^{2}$ \\ Akira Shouzu ${ }^{3}$ \\ Seitarou Omoto ${ }^{4}$ \\ Norihito Inami ${ }^{4}$ \\ Shinya Fujita' \\ Takeshi Tamaki' \\ Takashi Yokoi' \\ Toshiki Shimizu' \\ Tomoki Ito'}

'First Department of Internal Medicine, Kansai Medical University, Osaka, Japan; ${ }^{2}$ Department of Internal Medicine, Ueda Hospital, Osaka, Japan; ${ }^{3}$ Department of Internal Medicine, Saiseikai Izuo Hospital, Osaka, Japan; ${ }^{4}$ Second Department of Internal Medicine, Kansai Medical University, Osaka Japan
Correspondence: Shosaku Nomura First Department of Internal Medicine, Kansai Medical University 10-15, Fumizono-Cho, Moriguchi City,

Osaka, 570-8507, Japan

Tel +8I 669939453

Fax +8I 66992 I293

Email shosaku-n@mbp.ocn.ne.ip

\begin{abstract}
The effects of statins on two platelet activation markers, plasiminogen activator inhibitor (PAI)-1 and adiponectin, were investigated in 68 patients with hyperlipidemia. The patients were treated with pitavastatin with a dosage of $2 \mathrm{mg}$ daily. The plasma levels of platelet-derived microparticles (PDMP), soluble CD40 ligand (sCD40L), sP-selectin, PAI-1, and adiponectin were measured at baseline and after 6 months of treatment in both groups. In hyperlipidemic patients, the plasma levels were higher in PDMP, sCD40L, sP-selectin, and PAI-1, and lower in adiponectin, compared to the normolipidemic controls. Plasma PDMP and SCD40L were positively correlated, while plasma adiponectin was negatively correlated with the plasma levels of PAI-1. No significant differences were observed in the plasma levels of PDMP, sCD40L, sP-selectin, and PAI-1 before and after treatment. A significant increase in plasma adiponectin levels was observed after 6 months of treatment with pitavastatin. When the patients treated with pitavastatin were divided into two groups according to the adiponectin response to pitavastatin treatment, significant decreases in plasma PAI-1, PDMP, and SCD40L levels were observed after pitavastatin treatment in the responder group. These findings suggest that PDMP, sCD40L, and PAI-1 may participate in the development of atherothrombosis in patients with hyperlipidemia, and that pitavastatin may exert an adiponectin-dependent antiatherothrombotic effect in hyperlipidemic patients.
\end{abstract}

Keywords: hyperlipidemia, PAI-1, pitavastatin, adiponectin, atherothrombosis

\section{Introduction}

A high level of plasma in cholesterol is a risk factor for atherosclerosis, and high plasma levels of low-density lipoprotein cholesterol (LDL-C) promote the development of atherosclerotic disease. ${ }^{1-3}$ Since modified LDL (eg, by oxidation) is capable of loading macrophages with cholesterol, while unmodified LDL is not, ${ }^{4}$ oxidized LDL (oxLDL) is considered particularly atherogenic. Several interactions between the blood components of the coagulation and the fibrinolytic systems play an important role in the progression of atherosclerosis, ${ }^{5}$ and high levels of plasminogen activator inhibitor (PAI)-1 have been demonstrated in atherothrombosis. ${ }^{6}$ It has been shown that oxLDL induces PAI-1 expression in endothelial cells, ${ }^{7}$ and that this oxLDL-induced PAI-1 expression in endothelial cells plays an important role in the pathophysiology of atherothrombosis. ${ }^{8}$ For example, PAI-1 contributes to thrombin generation and thrombus formation by generating tissue factor. ${ }^{9}$ Therefore, PAI-1 ultimately causes vascular complications with the participation of the blood coagulation system.

Adiponectin, the most abundant adipose tissue-specific protein, is exclusively expressed in and secreted by the adipose tissue. ${ }^{10}$ Plasma adiponectin concentrations 
are known to decrease in obese individuals ${ }^{10,11}$ with type 2 diabetes $^{12}$ and to be closely related to whole-body insulin sensitivity. ${ }^{13}$ The protein occurs abundantly in circulation ${ }^{11}$ and stimulates nitric oxide production in vascular endothelial cells, which ameliorates the endothelial function. ${ }^{14,15}$ These observations suggest that the antiatherogenic properties of adiponectin may involve its nitric oxide-dependent antiplatelet effects.

Large clinical trials of 3-hydroxy-3-methylglutarylcoenzyme A (HMG-CoA) reductase inhibitors (statins) have shown that lowering plasma cholesterol is associated with decreasing incidents of cardiovascular events and a delay in the progression of atherosclerosis. ${ }^{16,17}$ Animal experiments have demonstrated that statins also inhibit PAI-1 production. ${ }^{18}$ Pitavastatin is an $\mathrm{HMG}-\mathrm{CoA}$ reductase inhibitor that significantly reduces the plasma levels of total cholesterol (TC), LDL-C, and triglycerides (TG), while causing modest elevation in the plasma high-density lipoprotein cholesterol (HDL-C). ${ }^{19,20}$ This statin has various pleiotropic effects on platelets, monocytes/macrophages, and endothelial cells. ${ }^{21-23}$ However, the effects of pitavastatin on the connections between activated platelets, PAI-1 and adiponectin, are poorly understood. This study investigates the effects of pitavastatin treatment on the plasma levels of platelet activation markers, PAI-1, and adiponectin, in hyperlipidemic patients. The aim of this study is to determine whether pitavastatin affects the plasma levels of PAI-1.

\section{Materials and methods Patients}

The study group included 50 normolipidemic controls and 81 hyperlipidemic patients. However, 13 patients dropped out of the study due to disease aggravation or the patient's removal. Therefore, 68 patients were analyzed for this study (Table 1; baseline data). Control patients were recruited from the hospital staff as well as other sources. From March 2004 to October 2009, hyperlipidemic patients were selected from the patients admitted to our hospitals. The study protocol was approved by the Institutional Review Board (IRB) of our institutions and written informed consent was obtained from each patient prior to the start of the trial. The participation criteria included the absence of a history of inflammatory, coronary artery, or cerebrovascular disease for 3 months prior to enrollment, as well as the absence of clinically detectable renal (serum creatinine $\geq 2.0 \mathrm{mg} / \mathrm{dL}$ ), hepatic (elevated serum transaminase), infectious (fever or elevated white blood cell count), or malignant disease (as determined by ultrasonography or computed tomography).
Table I Demographic and clinical characteristics of hyperlipidemic patients and normolipidemic controls

\begin{tabular}{llll}
\hline & $\begin{array}{l}\text { Normolipidemic } \\
\text { controls }\end{array}$ & $\begin{array}{l}\text { Hyperlipidemic } \\
\text { patients }\end{array}$ & P value \\
\hline $\mathrm{n}$ & 50 & 68 & \\
Men/women (no) & $33 / 17$ & $32 / 36$ & \\
Age (years) & $54 \pm \mathrm{II}$ & $62 \pm 10$ & $<0.0 \mathrm{I}$ \\
$\mathrm{BMI}\left(\mathrm{kg} / \mathrm{m}^{2}\right)$ & $24.1 \pm 3.7$ & $25.8 \pm 3.7$ & $\mathrm{NS}$ \\
$\mathrm{HbA}_{\mathrm{Ic}}(\%)$ & $6.1 \pm 2.7$ & $7.4 \pm 2.7$ & $<0.05$ \\
$\mathrm{TC}(\mathrm{mg} / \mathrm{dL})$ & $194 \pm 33$ & $249 \pm 42$ & $<0.00 \mathrm{I}$ \\
$\mathrm{LDL}-\mathrm{C}(\mathrm{mg} / \mathrm{dL})$ & $117 \pm 16$ & $155 \pm 30$ & $<0.00 \mathrm{I}$ \\
$\mathrm{DM}(\%)$ & 26.0 & 52.9 & \\
Smoking $(\%)$ & 20.0 & 23.5 & \\
$\mathrm{P}-\mathrm{CAD}(\%)$ & 6.0 & 13.2 & \\
$\mathrm{P}-\mathrm{CD}(\%)$ & 6.0 & 10.3 & \\
\hline
\end{tabular}

Notes: Data are shown as mean \pm SD. $P$ value, hyperlipidemic patients versus normolipidemic controls.

Abbreviations: $\mathrm{BMI}$, body mass index; $\mathrm{HbA}_{\mathrm{Ic}}$, hemoglobin $\mathrm{A}_{\mathrm{Ic}} ; \mathrm{TC}$, total cholesterol; LDL-C, low-density lipoprotein cholesterol; DM, diabetes mellitus; $\mathrm{P}-\mathrm{CAD}$, previous history of coronary artery disease; P-CD, previous history of cerebrovascular disease.

Other lipid-lowering agents were withheld, owing to their potential influence on the data interpretation. These medications were stopped at least 2 weeks prior to the initiation of pitavastatin or simvastatin therapy. Twenty-three patients received aspirin and 44 patients received an angiotensin II receptor blocker (ARB) in addition to the statin. Of the 68 hyperlipidemic patients, 36 had type 2 diabetes (Table 1); of these 36 , eleven were under treatment with sulfonylureas, 10 with $\alpha$-glucosidase inhibitors, and seven with insulin injections. Hyperlipidemia was defined in accordance with the Guidelines for Diagnosis and Treatment of Hyperlipidemia in Adults established by the Japan Atherosclerosis Society. ${ }^{24}$ Hyperlipidemia is defined as LDL-C greater than $140 \mathrm{mg} / \mathrm{dL}$, TG greater than $150 \mathrm{mg} / \mathrm{dL}$, or HDL-C less than $40 \mathrm{mg} / \mathrm{dL}$. LDL-C was calculated using the following formula: LDL-C $=\mathrm{TC}-(\mathrm{TG} / 5)-\mathrm{HDL}-\mathrm{C}$. Type 2 diabetes was defined in accordance with the criteria of the American Diabetes Association. ${ }^{25}$ Table 1 shows the clinical characteristics of the hyperlipidemic patients and control subjects.

\section{Study design}

Pitavastatin at $2 \mathrm{mg}$ /day (Kowa Company Ltd, Tokyo, Japan) was administered to the hyperlipidemic patients for 6 months. No other changes to the pharmacologic regimens of the patients were made during the course of the trial. In addition, changes in food habits, such as diet, were not recorded during this study. The clinical and biochemical data collected before and after 6 months of therapy with pitavastatin were analyzed. 


\section{Measurements of the plasma levels of platelet-derived microparticles, soluble factors, PAI-I, and adiponectin}

Fasting blood samples from patients and controls were collected from the peripheral veins into vacutainers containing EDTA-ACD (NIPRO Co, Ltd, Osaka, Japan) using 21-gauge needles to minimize platelet activation. The samples were gently mixed by inverting the tubes once or twice and were then kept at room temperature for a maximum period of 2-3 hours. Immediately after centrifugation at $8000 \mathrm{~g}$ for 5 minutes, $200 \mu \mathrm{L}$ of the upper-layer supernatant of the $2 \mathrm{~mL}$ samples was collected to avoid contamination by the platelets. The collected samples were stored at $-40^{\circ} \mathrm{C}$ until analysis. The platelet-derived microparticle (PDMP) levels were measured twice and the mean values were recorded. Furthermore, some basic studies were carried out prior to this assessment using clinical specimens. The enzyme-linked immunosorbent assay (ELISA) kit used for the PDMP measurement ${ }^{26,27}$ was obtained from JIMRO Co, Ltd (Tokyo, Japan). Soluble CD40 ligand (sCD40L) was measured with an ELISA kit purchased from Chemikon International Inc (Temecula, CA). Plasma sP-selectin and PAI-1 were measured with a monoclonal antibody-based ELISA kit purchased from Invitrogen International Inc (Camarillo, CA), while plasma adiponectin was measured with an Adiponectin ELISA kit purchased from Otsuka Pharmaceuticals Co, Ltd (Tokyo, Japan). The recombinant products and standard solutions provided with the commercial kits were used as positive controls in each assay. All the kits were used in accordance with the manufacturer's instructions.

\section{Statistical analysis}

The data were expressed as the mean \pm SD and analyzed by a two-factor analysis of variation (ANOVA) for repeated measures, as appropriate. Between-group comparisons were made using the Bonferroni test and within-group differences were determined by Student's $t$ - test for paired values. The correlation between the PAI-1 and after continuousresponse variables were assessed by a univariate and a multivariate linear regression analysis. $P$-values less than 0.05 were considered statistically significant.

\section{Results}

In hyperlipidemic patients, the plasma levels were higher in PDMP, sCD40L, sP-selectin, and PAI-1 and lower in adiponectin (Table 2), compared to the normolipidemic controls. For hyperlipidemic patients, univariate analysis
Table 2 Plasma levels of soluble factors, chemokines, and adiponectin in the normolipidemic controls and hyperlipidemic patients

\begin{tabular}{llll}
\hline & $\begin{array}{l}\text { Normolipidemic } \\
\text { control }\end{array}$ & $\begin{array}{l}\text { Hyperlipidemic } \\
\text { patients }\end{array}$ & P value \\
\hline $\mathrm{n}$ & 50 & 68 & \\
PDMP $(\mathrm{U} / \mathrm{mL})$ & $8.3 \pm 4.3$ & $10.7 \pm 4.5$ & $<0.05$ \\
sCD40L $(\mathrm{ng} / \mathrm{mL})$ & $9.2 \pm 3.7$ & $13.1 \pm 3.6$ & $<0.01$ \\
sP-selectin $(\mathrm{ng} / \mathrm{mL})$ & $147 \pm 35$ & $179 \pm 52$ & $<0.05$ \\
PAl-I $(\mathrm{ng} / \mathrm{mL})$ & $17.8 \pm 2.6$ & $29.6 \pm 5.1$ & $<0.01$ \\
Adiponectin $(\mu g / \mathrm{mL})$ & $4.63 \pm 1.55$ & $3.49 \pm 1.32$ & $<0.01$ \\
\hline
\end{tabular}

Notes: Data are shown as mean \pm SD. $P$ value, hyperlipidemic patients versus normolipidemic controls.

Abbreviations: PDMP, platelet-derived microparticle; sCD40L, soluble CD40 ligand; sP-selectin, soluble P-selectin; PAI-I, plasminogen activator inhibitor I.

showed that BMI, HDL-C, PDMP, sCD40L, sP-selectin, and adiponectin were significantly associated with PAI-1 (Table 3). In addition, PDMP, sCD40L, sP-selectin, and adiponectin were significant factors in the multivariate model with PAI-1 (Table 3).

No significant differences were observed in the plasma levels of PDMP, sCD40L, sP-selectin, or PAI-1 before and after pitavastatin administration (Table 4). On the other hand, plasma adiponectin levels significantly increased after 6 months of treatment with pitavastatin (Table 4).

We divided the patients of the pitavastatin group into two subgroups (responders and nonresponders) according to their adiponectin response to the pitavastatin treatment. The responders were defined as patients in whom plasma

Table 3 Univariate and multivariate analysis of PAI-I

\begin{tabular}{|c|c|c|c|c|}
\hline & \multicolumn{2}{|c|}{ Univariate } & \multicolumn{2}{|c|}{ Multivariate } \\
\hline & $\beta$ & $P$ value & $\beta$ & $P$ value \\
\hline Age (years) & 0.203 & 0.059 & 0.097 & 0.566 \\
\hline BMI & 0.221 & 0.053 & 0.155 & 0.202 \\
\hline $\mathrm{PLT}\left(\times 10^{4} / \mu \mathrm{L}\right)$ & 0.076 & $0.45 I$ & & \\
\hline 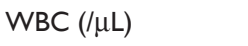 & 0.026 & 0.795 & & \\
\hline TC (mg/dL) & 0.041 & 0.625 & & \\
\hline HDL-C (mg/dL) & -0.263 & 0.031 & -0.239 & 0.065 \\
\hline TG (mg/dL) & 0.033 & 0.695 & & \\
\hline $\mathrm{HbA}_{\mathrm{lc}}(\%)$ & 0.136 & 0.172 & & \\
\hline BUN (mg/dL) & 0.135 & 0.208 & & \\
\hline CRTN (mg/dL) & 0.137 & 0.183 & & \\
\hline PDMP (U/mL) & 0.319 & $<0.00 I^{*}$ & 0.252 & 0.009 \\
\hline $\mathrm{sCD} 40 \mathrm{~L}(\mathrm{ng} / \mathrm{mL})$ & 0.281 & $<0.001 *$ & 0.212 & 0.044 \\
\hline sP-selectin (ng/mL) & 0.268 & $<0.00 I^{*}$ & 0.217 & 0.023 \\
\hline Adiponectin $(\mu \mathrm{g} / \mathrm{mL})$ & -0.272 & $<0.001 *$ & -0.215 & 0.049 \\
\hline
\end{tabular}

Note: $\beta$ is the standardized regression coefficient.

Abbreviations: PLT, platelet count; WBC, white blood cell; TC, total cholestrol; $\mathrm{HDL}-\mathrm{C}$, high density lipoprotein cholesterol; TG, triglycerides; $\mathrm{Hb}_{1 \mathrm{c}}$, hemoglobin $\mathrm{A}_{1 \mathrm{c}}$; $\mathrm{BUN}$, blood urea nitrogen; CRTN, creatinine; PDMP, platelet-derived microparticle; sCD40L, soluble CD40 ligand; sP-selectin, soluble P-selectin; PAI-I, plasminogen activator inhibitor $\mathrm{I}$. 
Table 4 Changes in the plasma levels of soluble factors, PAI-I, and adiponectin before and after pitavastatin treatment in hyperlipidemic patients

\begin{tabular}{llcl}
\hline & $\mathbf{0}$ & $\mathbf{6 M}$ & P value \\
\hline PDMP $(\mathrm{U} / \mathrm{mL})$ & $10.7 \pm 4.5$ & $9.8 \pm 4.6$ & NS \\
SCD40L $(\mathrm{ng} / \mathrm{mL})$ & $13.1 \pm 3.6$ & $12.8 \pm 3.5$ & NS \\
sP-selectin $(\mathrm{ng} / \mathrm{mL})$ & $179 \pm 52$ & $172 \pm 46$ & NS \\
PAI-I $(\mathrm{ng} / \mathrm{mL})$ & $29.1 \pm 5.1$ & $26.2 \pm 4.9$ & NS \\
Adiponectin $(\mu \mathrm{g} / \mathrm{mL})$ & $3.49 \pm 1.32$ & $4.36 \pm 1.29$ & $P<0.05$ \\
\hline
\end{tabular}

Notes: Data are shown as mean \pm SD. $P$ value, 0 versus 6 months. Abbreviations: PDMP, platelet-derived microparticle; sCD40L, soluble CD40 ligand; sP-selectin, soluble P-selectin; PAI-I, plasminogen activator inhibitor I; NS, not significant.

adiponectin levels increased by one-and-a-half times or more after pitavastatin treatment, compared to their pretreatment levels. As shown in Figure 1, LDL-C levels significantly decreased in the responder and nonresponder groups. However, there were no significant differences in LDL-C levels between the groups. The plasma PDMP, sCD40L, and PAI-1 levels significantly decreased in the responder group after pitavastatin treatment compared with those in the nonresponder group. There were no significant changes in plasma levels of BMI and sP-selectin after pitavastatin treatment in either the responder or the nonresponder group.

\section{Discussion}

PAI-1 has a significant effect on the occlusive thrombosis of atherosclerotic lesions, ${ }^{28}$ and endothelial and smooth muscle cells can produce PAI-1 that localizes in the atheroma. ${ }^{29,30}$ In addition, oxLDL was revealed to enhance the production of PAI-1 in endothelial and smooth muscle cells. ${ }^{7,31}$ These reports suggest that PAI-1 may play an important role in the development of atherosclerosis in hyperlipidemic patients. In fact, previous studies have demonstrated an association between the plasma levels of PAI-1 and the risk for future coronary events. ${ }^{32,33}$ The most interesting finding of this study was that PAI-1 plasma levels were negatively correlated with adiponectin plasma levels. Plasma adiponectin concentrations are known to decrease in obese individuals ${ }^{10}$ and are closely related to whole-body insulin sensitivity. ${ }^{13}$ Hotta et $\mathrm{al}^{12}$ report a significant decrease in adiponectin plasma concentrations in patients with type 2 diabetes. Furthermore, it has been reported that adiponectin inhibits monocyte adhesion to endothelial cells..$^{10,34,35}$ Therefore, it

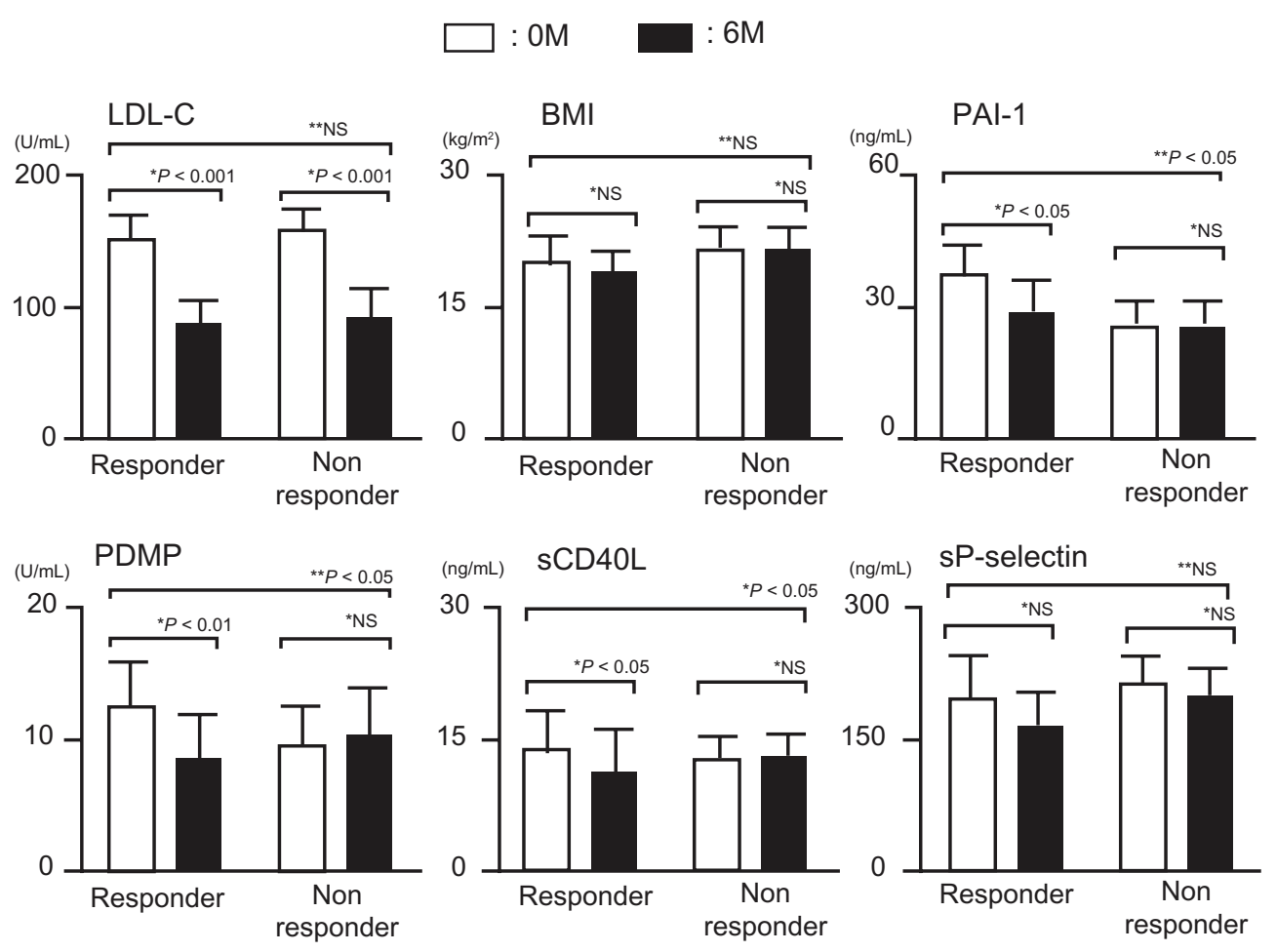

Figure I Changes in plasma LDL-C, BMI, PAI-I, PDMP, sCD40L, and sP-selectin levels in the two patient groups divided according to the adiponectin response to pitavastatin treatment. Responders were defined as patients showing an increase by one-and-a-half times or more of the plasma adiponectin concentrations after pitavastatin treatment, compared to pretreatment levels.

Notes: Bars are shown as mean $\pm \mathrm{SD}$. *Student's $t$-test (before versus after pitavastatin treatment); **ANOVA analysis (reponders versus nonresponders).

Abbreviations: LDL-C, low-density lipoprotein cholesterol; BMI, body mass index; PAI-I, plasminogen activator inhibitor; PDMP, platelet-derived microparticle; sCD40L, soluble CD40 ligand; sP-selectin, soluble P-selectin. 
is also possible that adiponectin inhibits the generation of monocyte or endothelial cell-derived tissue factor in which PAI-1 also participates. These reports support the negative correlation between the plasma levels of PAI-1 and adiponectin determined in this study. Hypoadiponectinemia is associated with endothelial dysfunction. ${ }^{36}$ In addition, hypoadiponectinemia also appears to cause platelet activation. Hypoadiponectinemia is also associated with decreased levels of nitric oxide, which regulates platelet activation, because adiponectin stimulates the production of nitric oxide in vascular endothelial cells ${ }^{14,15,36}$ and platelet activation is inhibited at low concentrations of nitric oxide. In the present study, the plasma levels of PDMP, sCD40L, and sP-selectin, which induce platelet activation, also significantly increased in the hyperlipidemic patients. Therefore, it appears that a close connection exists between platelet activation, PAI-1, and adiponectin in patients with hyperlipidemia.

Statins play a critical role in cholesterol metabolism. ${ }^{37}$ Statins also have pleiotropic effects, such as antithrombotic and anti-inflammatory effects. ${ }^{38}$ Oxidative stress plays a pivotal role in the pathogenesis of atherosclerosis. ${ }^{38}$ In other words, inflammation is closely associated with the formation of reactive oxygen species (ROS), and ROS activates redoxsensitive genes such as matrix metalloproteinase, intercellular adhesion molecule 1, and PAI-1, resulting in the development of atherosclerosis. ${ }^{38,39}$ Statins possess such antiredox effects. ${ }^{39-41}$ In particular, lovastatin has been shown to inhibit oxLDL-induced PAI-1 elevation. ${ }^{42}$ However, this study did not observe a significant reduction in PAI-1 plasma levels in the pitavastatin-treated group either. We previously reported that pitavastatin could cause an elevation of adiponectin in hyperlipidemic patients with type 2 diabetes. ${ }^{43,44}$ In these reports, we inferred two possibilities about this mechanism including the inhibition of production/activity of ROS and an influence on adipocyte physiology via the effects on the sterol regulatory element binding the proteins- $1 \mathrm{c} .{ }^{45}$ In addition, we found that pitavastatin caused the adiponectin-dependent reduction of sE-selectin and sL-selectin. ${ }^{44}$ However, we did not determine a correlation between adiponectin and PAI-1 in previous studies. Nevertheless, when the patients of the pitavastatin group were divided into two subgroups according to the adiponectin response to pitavastatin treatment, a significant decrease in the plasma levels of PAI-1 was found after pitavastatin treatment in the adiponectin responder group. In addition, similar results were found for the PDMP and SCD40L plasma. These results are of interest because reducing PAI-1 plasma plays a potential role in the reduction of the risk of atherothrombosis in hyperlipidemic patients. In addition, the PAI-1 plasma was negatively correlated with adiponectin plasma. These results suggest that pitavastatin causes adiponectin-dependent improvement of the plasma levels of PAI-1, PDMP, and SCD40L in hyperlipidemic patients. We believe that the effects of pitavastatin on PAI-1 activity depend on adiponectin. Therefore, pitavastatin could inhibit the progression of atherothrombosis by decreasing the plasma levels of LDL-C and promoting adiponectin-dependent improvement of the PAI-1, PDMP, and SCD40L plasma.

\section{Acknowledgments}

This study was supported in part by a grant from the Japan Foundation of Neuropsychiatry and Hematology Research, the Research Grant for Advanced Medical Care from the Ministry of Health and Welfare of Japan, and a grant (13670760 to SN) from the Ministry of Education, Science and Culture of Japan.

\section{Disclosure}

The authors do not have any conflicts of interest to report in this work.

\section{References}

1. Lusis AJ. Atherosclerosis. Nature. 2000;407:233-241.

2. Croft KD, Beilin LJ, Vandongen R, Rouse I, Masarei J. Leukocyte and platelet function and eicosanoid production in subjects with hypercholesterolemia. Atherosclerosis. 1990;83:101-109.

3. Chobanian AV. Single risk factor intervention may be inadequate to inhibit atherosclerosis progression when hypertension and hypercholesterolemia co-exist. Hypertension. 1991;18:130-131.

4. Fogelman A, Schechter I, Seager J, et al. Malondialdehyde alteration of low density lipoproteins leads to chelesterylester accumulation in macrophages. Proc Natl Acad Sci U S A. 1980;77:2214-2218.

5. Rabbani LE, Loscalzo J. Recent observations on the role of hemostatic determinants in the development of the atherosclerotic plaque. Atherosclerosis. 1994;105:1-7.

6. Sobel BE, Taatjes DJ, Schneider DJ. Intramural plasminogen activator inhibitor type-1 and coronary atherosclerosis. Artherioscler Thromb Vasc Biol. 2003;23:1979-1989.

7. Zhao R, Ma X, Xie X, Shen GX. Involvement of NADPH oxidase in oxidized LDL-induced upregulation of heat shock factor-1 and plasminogen activator inhibitor-1 in vascular endothelial cells. Am J Physiol Endocrino Metab. 2009;297:E104-E111.

8. Kuniyasu A, Tokunaga M, Yamamoto T, et al. Oxidized LDL and lysophosphatidylcholine stimulate plasminogen activator inhibitor-1 expression through reactive oxygen species generation and ERK $1 / 2$ activation in 3T3-L1 adipocytes. Biochim Biophys Acta. 2011;1811:153-162.

9. Shen GX. Vascular cell-derived fibrinolytic regulators and atherothrombotic vascular disorders. Int J Mol Med. 1998;1:399-408.

10. Ouchi N, Kihara S, Arita Y, et al. Adiponectin, an adipocyte- derived plasma protein, inhibits endothelial NF-kappa B signaling through a cAMP-dependent pathway. Circulation. 2000;102:1296-1301.

11. Arita Y, Kihara S, Ouchi N, et al. Paradoxical decrease of an adiposespecific protein, adiponectin, in obesity. Biochem Biophys Res Commun. 1999;257:79-83.

12. Hotta K, Funahashi T, Arita Y, et al. Plasma concentrations of a novel, adipose-specific protein, adiponectin, in type 2 diabetes patients. Artherioscler Thromb Vasc Biol. 2000;20:1595-1599. 
13. Weyer C, Funahashi T, Tanaka S, et al. Hypoadiponectinemia in obesity and type 2 diabetes: close association with insulin resistance and hyperinsulinemia. J Clin Endocrinol Metab. 2001;86:1930-1935.

14. Chen H, Montagnani M, Funahashi T, Shimomura I, Quon MJ. Adiponectin stimulates production of nitric oxide in vascular endothelial cells. J Biol Chem. 2003;278:45021-45026.

15. Hattori Y, Suzuki M, Hattori S, Kasai K. Globular adiponectin upregulates nitric oxide production in vascular endothelial cells. Diabetologia. 2003;46:1543-1549.

16. Scandinavian Simbvastatin Survival Study Group. Randomised trial of cholesterol lowering in 4444 patients with coronary heart disease: the Scandinavian Simvastatin Survival Study (4S). Lancet. 1994;344:1383-1389.

17. West of Scotland Coronary Prevention Group. Influence of pravastatin and plasma lipids on clinical events in the West of Scotland Coronary Prevention Study (WOSCOPS). Circulation. 1998;97:1440-1445.

18. Ishihara Y, Ohmori K, Mizukawa M, et al. Beneficial direct adipotropic actions of pitavastatin in vitro and their manifestations in obese mice. Atherosclerosis. 2010;212:131-138.

19. Noji Y, Higashikata T, Inazu A, et al. Long-term treatment with pitavastatin (NK-104), a new HMG-CoA reductase inhibitor, in patients with heterozygous familial hypercholesterolemia. Atherosclerosis. 2002;163:157-164.

20. Saito Y, Yamada N, Teramoto T, et al. Clinical efficacy of pitavastatin, a new 3-hydroxy- 3-methylglutaryl coenzyme A reductase inhibitor, in patients with hyperlipidemia: dose-finding study using the double-bind, three-group parallel comparison. Arzeimittelforschung. 2002;52:251-255.

21. Han J, Zhou X, Yokoyama T, et al. Pitavastatin downregulates expression of the macrophage type B scavenger receptor, CD36. Circulation. 2004;109:790-796.

22. Kawakami A, Tani M, Chiba T, et al. Pitavastatin inhibits remnant lipoprotein-induced macrophage foam cell formation through apoB48 receptor-dependent mechanism. Artherioscler Thromb Vasc Biol. 2005;25:424-429.

23. Wang J, Tokoro T, Matsui K, Higa S, Kitajima J. Pitavastatin at low dose activates endothelial nitric oxide synthase through PI3K-AKT pathway in endothelial cells. Life Scien. 2005;76:2257-2268.

24. The Expert Committee on the Guideline for Diagnosis and Treatment of Hyperlipidemia. Investigating Committee of Guideline for Diagnosis and Treatment of Hyperlipidemia, Japan Atherosclerosis Society (JAS): Guideline for Diagnosis and Treatment of Hyperlipidemias in Adults. Doumyakukouka. 1997;25:1-34.

25. The Expert Committee on the Diagnosis and Classification of Diabetes Mellitus (ECDCDM). Report of the expert commitee on the diagnosis and classification of diabetes mellitus. Diabetes Care. 2003; (26 Suppl 1):S5-S20.

26. Osumi K, Ozeki Y, Saito S, et al. Development and assessment of enzyme immunoassay for platelet-derived microparticles. Thromb Haemost. 2001;85:326-330.

27. Nomura S, Uehata S, Saito S, et al. Enzyme immunoassay detection of platelet-derived microparticles and RANTES in acute coronary syndrome. Thromb Haemost. 2003;89:506-512.

28. Juhan-Vague I, Alessi MC, Vague P. Increased plasma plasminogen activator inhibitor 1 levels: a possible link between insulin resistance and atherothrombosis. Diabetologia. 1991;34:457-462.

29. Carmelier P, Moons L, Lijnen R, et al. Inhibitory role of plasminogen activator inhibitor-1 in arterial healing and neointima formation: a gene targeting and gene transfer study in mice. Circulation. 1997;96:3180-3191.
30. Ma GM, Halayko AJ, Stelmack GL, et al. Effects of oxidized and glycated low-density lipoprotections on transcription and secretion of plasminogen activator inhibitor-1 in vascular endothelial cells. Cardiovasc Pathol. 2006;15:3-10.

31. Dichtl W, Stiko A, Eriksson P, et al. Oxidized LDL and lysophosphatidylcholine stimulate plasminogen activator inhibitor-1 expression in vascular smooth muscle cells. Artherioscler Thromb Vasc Biol. 1999;19:3025-3032.

32. Brodsky SV, Malinowski K, Golightly M, Jesty J, Goligorsly MS. Plasminogen activator inhibitor-1 promotes formation of endothelial microparticles with procoagulant potential. Circulation. 2002;106: 2372-2378.

33. Lautamäki R, Rönnemaa T, Huupponen R, et al. Low serum adiponectin is associated with high circulating oxidized low-density lipoprotein in patients with type 2 diabetes mellitus and coronary artery disease. Metabolism. 2007;56:881-886.

34. Ouchi N, Kihara S, Arita Y, et al. Adipocyte-derived plasma protein, adiponectin, suppresses lipid accumulation and class A scavenger receptor expression in human monocyte-derived macropages. Circulation. 2001;103:1057-1063.

35. Okamoto Y, Arita Y, Nishida M, et al. An adipocyte-derived plasma protein, adiponectin, adheres to injured vascular walls. Horm Metab Res. 2000;32:47-50.

36. Shimabukuro M, Higa N, Asahi T, et al. Hypoadiponectinemia is closely linked to endothelial dysfunction in man. J Clin Endocrinol Metab. 2003;88:3236-3240.

37. Kureishi Y, Luo Z, Shiojima I, et al. The HMG-CoA reductase inhibitor simvastatin activates the protein kinase Akt and promotes angiogenesis in normocholesterolemic animals. Nat Med. 2000;6:1004-1010.

38. Brownlee M. Biochemistry and molecular cell biology of diabetic complications. Nature. 2006;414:813-820.

39. Stoll LL, McCormick ML, Denning GM, Weintraub NL. Antioxidant effects of statins. Drug Today. 2004;40:975-990.

40. Zeng L, Xu H, Chew TL, et al. Simvastatin modulates angiotensin II signaling pathway by preventing Rac 1-mediated upregulation of $\mathrm{p} 27$. J Am Soc Nephrol. 2004;15:1711-1720.

41. Hogue JC, Lamarche B, Tremblay AJ, et al. Differntial effect of atorvastatin and fenofibrate on plasma oxidized low-density lipoprotein, inflammation markers, and cell adhesion molecules in patients with type 2 diabetes mellitus. Metabolism. 2008;57:380-386.

42. Song CY, Kim BC, Lee HS. Lovastatin inhibits oxidized low-density lipoprotein-induced plasminogen activator inhibitor and transforming groth factor- $\beta_{1}$ expression via a decrease in Ras/extracellular signal-regulated kinase activity in mesangial cells. Transl Res. 2008; 151:27-35.

43. Inami N, Nomura S, Shouzu A, et al. Effects of pitavastatin on adiponectin in patients with hyperlipidemia. Pathophysiol Haemost Thromb. 2007-2008;36:1-8.

44. Nomura S, Shouzu A, Omoto S, et al. Correlation between adiponectin and reduction of cell adhesion molecules after pitavastatin treatment in hyperlipidemic patients with type 2 diabetes mellitus. Thromb Res. 2008;122:39-45.

45. Erol A. The role of fat tissue in the cholesterol lowering and the pleiotropic effects of statins - statins activate the generation of metabolically more capable adipocytes. Med Hypotheses. 2005;64:69-73.
International Journal of General Medicine

\section{Publish your work in this journal}

The International Journal of General Medicine is an international, peer-reviewed open-access journal that focuses on general and internal medicine, pathogenesis, epidemiology, diagnosis, monitoring and treatment protocols. The journal is characterized by the rapid reporting of reviews, original research and clinical studies across all disease areas.

\section{Dovepress}

A key focus is the elucidation of disease processes and management protocols resulting in improved outcomes for the patient. The manuscript management system is completely online and includes a very quick and fair peer-review system. Visit http://www.dovepress.com/ testimonials.php to read real quotes from published authors. 